\title{
APÉNDICE
}

\section{ESTUDIO MINERALÓGICO DE UNAS TERRACOTAS DEL TEMPLO ROMANO DE LA ENCARNACIÓN (CARAVACA, MURCIA)}

\author{
POR \\ R. ARANA CASTILLO \\ Departamento de Quimica Agrícola, Geologia y Edafologia \\ Facultad de Quimica Universidad de Murcia
}

\section{RESUMEN}

En este trabajo se estudian por métodos de rayos $\mathrm{X}$, análisis espectroquimico y microscopia de luz transmitida cuatro muestras de cerámicas del templo romano de La Encarnación (Caravaca, SE. de España) con objeto de obtener información sobre su composición mineralógica, aspectos texturales y probable fuente de esos materiales.

El estudio mineralógico revela en dos muestras una composición particular de los fragmentos diseminados (presencia de nódulos anortosíticos) que apoya la hipótesis de que esos materiales fueron importados.

\section{SUMMARY}

Four characteristic samples of ceramics belonging to Roman temple of La Encarnación (Caravaca, SE. Spain) are studied in this work by X-Ray methods, spectrochemical analysis and transmitted light microscopy in order to achieve several data on their mineralogical composition, textural aspects and also on the probable source of these materials.

The mineralogical study reveals in two samples a particular composition of the disseminated fragments (presence of anorthositic nodules) that support the hypothesis that these materials were imported.

\section{INTRODUCCIÓN}

Desde las últimas décadas se han venido aplicando con eficacia diversas técnicas mineralógicas al estudio de fragmentos cerámicos, tanto antiguos como actuales, para obtener una información precisa sobre las materias primas originales y los procesos de elaboración. En este sentido, la Mineralogía puede ofrecer un complemento importante en una investigación esencialmente arqueológica. Aparte de la difracción y la fluorescencia de rayos $\mathrm{X}$, los métodos ópticos por luz transmitida y reflejada, microscopía electrónica y de barrido (scanning), técnicas térmicas, análisis químico, espectroscopia Mössbauer e infrarroja y otros métodos complementarios, son de uso cada vez más frecuente (Jourdain, 1966; Balcázar et al., 1983; Arana, 1985; García Ramos et al., 1986; De Andrés et al., 1988, 1989; Bennett y Oliver, 1992, entre otros). 
En la fabricación de un producto cerámico intervienen numerosos factores según las propiedades deseadas, el uso a que va destinado y la calidad que se pretende alcanzar. Así, tanto la composición mineralógica del material de partida, como el tipo y abundancia de desgrasantes, densidad de la pasta, tiempo y temperatura de cocción, pigmentos, grosor y tipo de barnices. etc., son parámetros que, bien de una manera empirica o mediante un control técnico adecuado, se pueden variar en un amplio margen, con lo que el producto final tendrá unas propiedades determinadas (Singer y Singer, 1979). En conjunto se producen una serie de transformaciones fisicas y químicas en el material inicial para dar un producto cerámico con unas propiedades totalmente nuevas.

Dentro del análisis integral del templo romano de La Encarnación se han seleccionado dos fragmentos de terracotas arquitectónicas utilizadas como motivos ornamentales ( $n .{ }^{\circ} 1$ y 4$)$ y otros dos de uso cultual ( n. $^{\circ} 2$ y 3 ) que destacan por la originalidad de las formas, con objeto de llevar a cabo un estudio mineralógico y obtener información sobre los procesos de fabricación de las cerámicas respectivas y sobre la posible fuente de partida para esos materiales.

En este sentido se ha efectuado un análisis de las muestras por microscopía de luz transmitida $\mathrm{y}$ un estudio por difracción y fluorescencia de rayos $\mathrm{X}$ para conocer la naturaleza de las fases representadas y algunos datos sobre el quimismo global en cuanto a elementos mayoritarios y trazas. Asimismo, se molió una pequeña cantidad de cada muestra y se seleccionó la fracción comprendida entre 125 y $250 \mu$ para efectuar una separación magnética de las particulas mediante un separador isodinámico Franz L-1. Las fracciones obtenidas (ferromagnéti$\mathrm{ca}$, paramagnética y diamagnética) se analizaron posteriormente por difracción de rayos $\mathrm{X}$ para conocer la identidad de las diferentes fases.

Este trabajo forma parte de un estudio mineralógico más completo sobre los diversos materiales empleados en la construcción del templo, su procedencia y los principales procesos de alteración que han actuado sobre ellos. De esta forma, las observaciones recogidas pueden encajarse en el contexto multidisciplinar con que se ha abordado la investigación de este interesante templo.

\section{ESTUDIO MICROSCÓPICO}

\section{Muestra $n .{ }^{\circ 1}$. Placa de revestimiento}

Se trata de una cerámica basta de tonos pardos, muy porosa, formada por una matriz micro a criptocristalina, con numerosas cavidades libres, tanto redondeadas como alargadas y generalmente orientadas. Los componentes que se pueden caracterizar ópticamente en la cerámica comprenden dos grupos bien definidos: de una parte, fragmentos líticos y de otra granos minerales dispersos en la trama, esencialmente cuarzo, carbonatos, feldespatos, piroxenos y menas metálicas.

Los fragmentos líticos corresponden a rocas de naturaleza muy desigual (cuarcitas y anortositas) y aparecen claramente individualizados de la trama. Los fragmentos de cuarcita son los más abundantes, con formas angulosas más o menos deformadas, bordes redondeados y contornos suaves, muy bien definidos. Están constituidos por un mosaico de pequeños cristales xenomorfos de cuarzo con orientación óptica muy variable, junto a pequeños haces de moscovita que definen claramente una esquistosidad de flujo. Contienen algunos granos intersticiales de clorita y feldespato potásico y todo el conjunto aparece fuertemente impregnado por óxidos y oxi-hidróxidos de hierro en un entramado muy compacto. 
Los fragmentos de anortosita están constituidos por un agregado de plagioclasa cálcica en cristales alargados, maclados y parcialmente interpenetrados con secciones prismáticas y basales de piroxeno de tipo augita, cuyos bordes se muestran parcialmente alterados. En conjunto, los cristales de plagioclasa y piroxeno aparecen fuertemente imbricados, con abundantes menas metálicas intersticiales, probablemente de tipo ilmenita $\mathrm{y} / \mathrm{o}$ hematites.

Los cristales dispersos en la trama corresponden a cuarzo, plagioclasa, piroxeno, calcita y mica.

Los cristales de cuarzo se caracterizan por una marcada heterometria de grano, desde algunos milimetros a varias micras y contornos irregulares. Es un componente muy abundante y el principal desgrasante de la cerámica.

Los fragmentos de carbonato presentan igualmente una marcada gradación en el tamaño de grano, con una alteración apreciable. Su naturaleza es calcítica y siguen a cuarzo en cuanto a abundancia relativa de los fragmentos clásticos.

Los cristales sueltos de piroxeno son de la misma naturaleza que los que aparecen en los fragmentos de anortosita y destacan de la trama por su fuerte refringencia y sus contornos idiomorfos a subidiomorfos.

La plagioclasa se encuentra en cristales alargados, con maclas polisintéticas en haces parcialmente interpenetrados y un tamaño apreciable, a veces superior a 0.5 milimetros.

Los cristales de mica aparecen dispersos en la matriz en forma de finos haces; corresponden a una moscovita.

Todo el conjunto aparece impregnado por una coloración pardo-rojiza, tanto en el interior de los fragmentos líticos como entre los componentes de la trama.

La figura 19a ofrece un aspecto general de esta cerámica, destacando la marcada heterometría de grano, tanto de los fragmentos líticos como de los cristales dispersos en la trama.

\section{Muestra n. ${ }^{\circ}$ 2. Pebetero}

Se trata de una cerámica parda de matriz microcristalina y una porosidad muy elevada, con abundantes cavidades de forma elipsoidal, subesférica o bien alargadas, producidas durante la etapa de cocción de la pasta original. En conjunto, tanto por la textura como por la composición, esta muestra es sensiblemente distinta a la anterior, particularmente por la ausencia de fragmentos de cuarcita y anortosita.

La cerámica empleada en el pebetero presenta una impregnación generalizada de óxidos de hierro que afecta a los distintos componentes, con escasas menas metálicas diseminadas y de pequeño tamaño, hecho que destaca al observar la muestra con un solo polarizador. Las principales fases cristalinas son las siguientes:

- Cuarzo, en cristales sueltos, subredondeados y angulosos, con una marcada gradación de tamaño, desde algunos milímetros a varias micras, por lo que es también un componente importante de la matriz. Algunos granos aparecen fragmentados, con microfisuras irregulares y contienen inclusiones aciculares de moscovita; otras inclusiones son de rutilo y hematites.

- Biotita, en pequeños haces parcialmente alterados y con un marcado pleocroismo en tonos pardo-rojizos; presenta una amplia gama de tamaño de grano, pasando sensiblemente a la matriz. También se encuentran cristales aciculares sueltos de moscovita, mucho más pequeños.

- Clorita, en agregados hojosos con débil pleocroismo en tonos verde-amarillentos. 
Ortosa. en cristales euhedricos con macla de karlsbad y con un tamaño entre 0.5 y $1 \mathrm{mi}$ limetros

- Calcita, en pequeñas secciones romboidales ocupando intersticios de la trama.

Anfibol de la serie tremolita-actinolita, en secciones tabulares y prismáticas con excelente exfoliacion.

Plagioclasa sodo-cálcica (oligoclasa-andesina), en pequeños agregados de cristales maclados segun la ley de la albita.

En la figura 19b se ofrece un aspecto general de esta muestra entre polarizadores cruzados. Destaca la elevada porosidad y la marcada heterometría de grano de sus componentes, fuertemente impregnados por óxidos de hierro, con predominio de tonos grises y negros.

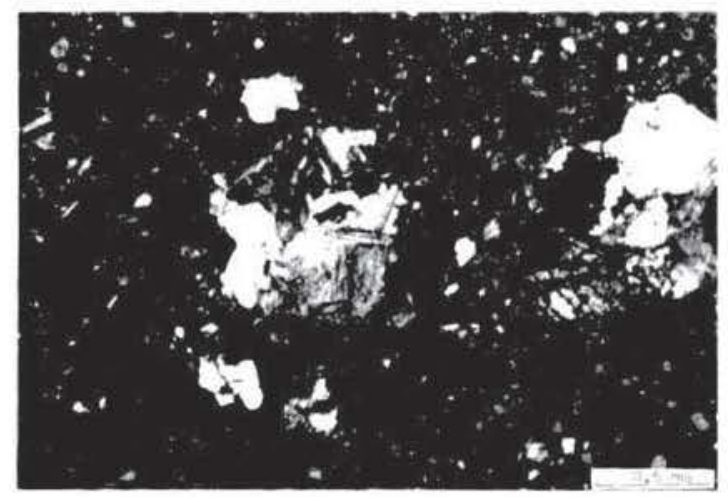

a)

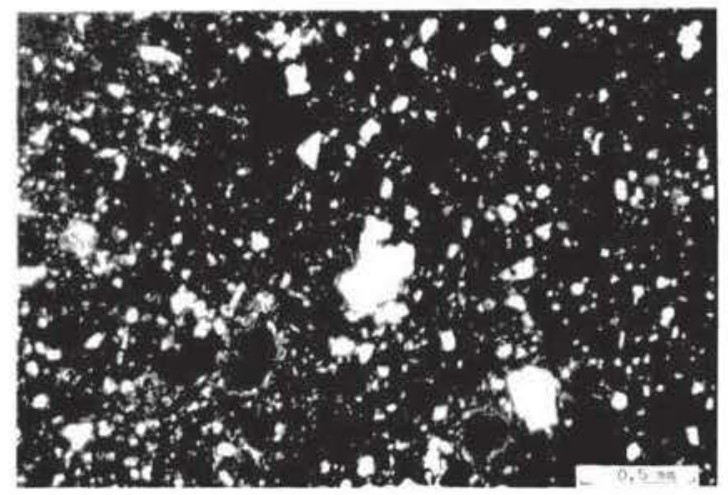

()

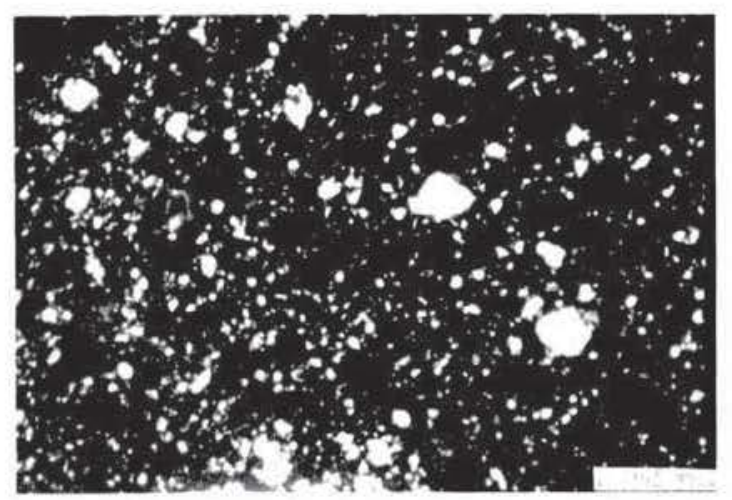

h)

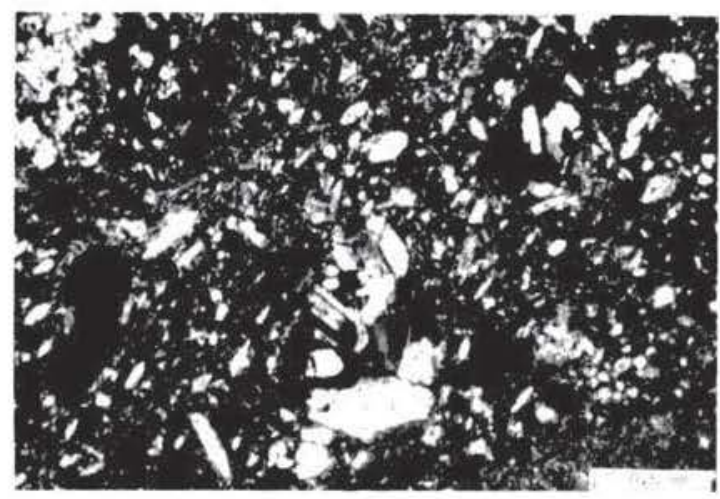

d)

Figura 19. - a) Placa de revestimiento, observada en luz transmitida y polarizadores cruzados. Destaca la presencia de fragmentos de anortosita y una marcada heterometría de grano en los componentes de la trama. b) Pebetero, en luz transmitida y polarizadores cruzados. Destaca la elevada porosidad de la muestra y la gran variación en el tamaño de grano. En tonos claros, cristales de cuarzo y calcita. c) Exvoto, en luz transmitida y polarizadores cruzados. Fuerte impregnación de óxidos de hierro entre los componentes de la trama, principalmente cuarzo y calcita, en tonos claros. d) Antefija, en luz transmitida y polarizadores cruzados. Se observan fenocristales maclados de plagioclasa cálcica, granos irregulares de cuarzo y una fuerte impregnación de óxidos de hierro. 


\section{Muestra n. ${ }^{\circ}$ 3. Exvoto}

Corresponde a una cerámica de tonos pardo-rojizos y matriz microcristalina, caracterizada por una elevada porosidad y una marcada heterometria de grano.

Sus principales rasgos son parecidos a los de la muestra anterior, aunque las proporciones de calcita y cuarzo están aquí ligeramente invertidas, siendo algo más abundante la primera.

Los principales componentes identificables al microscopio son los siguientes:

- Cuarzo, en cristales de bordes angulosos con una acusada gradación de tamaño. Algunos cristales presentan una microfisuración y pequeñas inclusiones de menas metálicas.

- Fragmentos angulosos de calcita, constituidos por agregados muy finos entre los cuales aparecen numerosas cavidades libres. Color de interferencia muy elevado.

- Haces de cristales de biotita con fuerte pleocroismo en tonos pardo-rojizos dispersos en la trama; generalmente son de pequeño tamaño y pasan también a la matriz. También se encuentran dispersos cristales aciculares de moscovita, menos abundante que la anterior.

- Plagioclasa sodo-cálcica (oligoclasa-andesina), en agregados maclados.

- Fragmentos angulosos de cuarcita, constituidos por cristales xenomorfos de cuarzo, con haces de moscovita y diseminaciones de menas metálicas.

Finalmente se debe destacar la impregnación generalizada de óxidos de hierro en los distintos componentes de la muestra y en los espacios intergranulares (figura 19c).

\section{Muestra $n .^{\circ}$ 4. Antefija}

Esta muestra es muy parecida a la $n .^{\circ} 1$ (placa de revestimiento). Se trata de una cerámica de grano grueso con abundantes restos líticos y numerosas cavidades vesiculares; también presenta una fuerte impregnación de óxidos de hierro entre los componentes y una diseminación de menas metálicas (magnetita, hematites y goethita).

La matriz es microcristalina, de un color rojizo característico, dentro de la cual destacan cristales de cuarzo, plagioclasa, fragmentos de cuarcita, piroxeno augítico, anfibol, calcita y micas (biotita y moscovita). Los rasgos son similares a los descritos para estos componentes en la muestra número 1. En la figura $19 \mathrm{~d}$ se ofrece un aspecto general de esta muestra entre polarizadores cruzados. Se advierten fenocristales de plagioclasa maclada, granos de bordes irregulares de cuarzo y una intensa impregnación de óxidos de hierro.

\section{ESTUDIO POR DIFRACCIÓN DE RAYOS X}

Se ha efectuado un rodaje de cada muestra en placas de polvo con objeto de identificar las diversas fases presentes. Asimismo, se han estudiado por esta técnica las fracciones ferromagnética, paramagnética y diamagnética en el tamaño comprendido entre 125 y 250 micras, obtenidas con un separador isodinámico. De esta forma se ha podido llevar a cabo una identificación más fácil de los minerales correspondientes a cada fracción, con un interés particular en el caso de las fases ferromagnéticas. 
En la Tabla I se indican las proporciones relativas de los minerales dominantes en cada muestra.

\begin{tabular}{|c|c|c|c|c|}
\hline & Placa & Pebetero & Exvoto & Antefija \\
\hline Q & $\mathrm{MA}$ & $\mathrm{MA}$ & $\mathrm{MA}$ & $\mathrm{MA}$ \\
\hline $\mathrm{Cc}$ & A & $\mathrm{E}$ & M & $\mathrm{MA}$ \\
\hline PI & E & $\mathrm{Tr}$ & $\mathrm{E}$ & $\mathrm{E}$ \\
\hline $\mathrm{FdK}$ & E & E & - & $\mathrm{E}$ \\
\hline Mica & $\mathrm{Tr}$ & E & $\mathrm{E}$ & $\mathrm{Tr}$ \\
\hline Clo & - & $\mathrm{Tr}$ & - & $\mathrm{Tr}$ \\
\hline Py & E & - & $\mathrm{Tr}$ & $\mathrm{Tr}$ \\
\hline Anf & - & $\mathrm{E}$ & $\mathrm{Tr}$ & - \\
\hline Hem & $\mathrm{Tr}$ & $\mathrm{Tr}$ & $\mathrm{Tr}$ & $\mathrm{Tr}$ \\
\hline Goe & $\mathrm{E}$ & $\mathrm{E}$ & $\operatorname{Tr}$ & $\mathrm{Tr}$ \\
\hline $11 \mathrm{~m}$ & $\mathrm{Tr}$ & - & - & $\mathrm{Tr}$ \\
\hline Mt & $\mathrm{Tr}$ & $\mathrm{Tr}$ & $\mathrm{Tr}$ & $\mathrm{Tr}$ \\
\hline
\end{tabular}

$\mathrm{Q}$, cuarzo; Cc, calcita; PI, plagioclasa; Mica, moscovita y/o biotita; Clo, clorita; Py, piroxeno (augita); Anf, anfibol (serie tremolita-actinolita); Hem, hematites; Goe, goethita; Ilm, ilmenita; Mt, magnetita. MA, muy abundante; $\mathrm{A}$, abundante; $\mathrm{M}$, medio; E, escaso; Tr, trazas; -, ausente.

Tabla I. Composición mineralógica de las cerámicas

El estudio difractométrico de las fracciones magnéticas confirma la presencia de magnetita como fase ferromagnética presente en todas las muestras aunque sólo al estado de trazas. La fracción paramagnética comprende los componentes más característicos de las menas metálicas diseminadas (hematites, ilmenita y goethita) junto a otros granos de la trama como piroxeno, anfibol y clorita. Finalmente, la fracción diamagnética es la más abundante y está formada por cuarzo, calcita, feldespatos y moscovita.

En el estudio de la muestra integra por fluorescencia de rayos $\mathrm{X}$ aparecen con gran intensidad las lineas características correspondientes a potasio, hierro y calcio; con intensidad media las de titanio y manganeso, junto a trazas de bario, cobalto, níquel, cromo, cobre, cinc, estroncio, rubidio, plomo y arsénico. La procedencia de estos elementos se puede relacionar fácilmente con los minerales anteriormente descritos. En efecto, el calcio forma parte de la red de la plagioclasa, piroxeno, anfibol y calcita; el potasio se relaciona con el feldespato potásico (ortosa) y micas. Los elementos hierro, titanio y manganeso van asociados a las menas metálicas diseminadas en la trama y en las impregnaciones en torno a los cristales. Los elementos pesados $(\mathrm{Zn}, \mathrm{Cu}, \mathrm{Ni}, \mathrm{Co}, \mathrm{Pb}$ y $\mathrm{As})$ están conectados igualmente con las menas metálicas citadas. Finalmente, el $\mathrm{Sr}$ debe ir asociado con $\mathrm{Ca}$ y el $\mathrm{Rb}$ con $\mathrm{K}$. 
No se observan diferencias cualitativas apreciables en la concentración de los elementos reseñados en las distintas muestras, aunque si se encuentran algunas variaciones en la intensidad de las lineas; asi, el Mn y Sr son más abundantes en las muestras 1 y 4 que en las 2 y 3 , confirmándose de esta forma un mayor parecido entre las dos primeras ( 1 y 4 ) y entre las dos últimas ( 2 y 3$)$.

\section{CONSIDERACIONES GENÉTICAS}

Aparte de conocer los rasgos esenciales sobre la textura y composición de un material cerámico, el estudio mineralógico trata de conseguir información sobre las condiciones en que se ha formado el producto y si existe una relación entre las materias primas utilizadas y las que se encuentran en un entorno próximo. De esta forma se puede establecer una hipótesis más o menos razonada sobre la procedencia de los materiales utilizados en su elaboración.

Durante el proceso de cocción se produce una serie de transformaciones químicas y mineralógicas en los productos originales que llegan a modificar totalmente su naturaleza. La materia prima normalmente está constituida por cantidades variables de los siguientes materiales: a) arcillas (caolinita, montmorillonita, ilita, clorita, etc.); $b$ ) sílice cristalina, generalmente en forma de cuarzo; $c$ ) pigmentos colorantes, sobre todo óxidos de hierro; $d$ ) carbonatos (calcita y/o dolomita); $e$ ) feldespatos alcalinos (ortosa, albita, etc.) y $f$ ) materia orgánica y compuestos diversos, generalmente incorporados con fragmentos de rocas.

Las transformaciones químicas se inician con una pérdida gradual de agua en sus diversas formas estructurales (higroscópica, de constitución y ceolítica) junto a procesos de transformación de las diversas fases presentes en función de la temperatura. Los principales procesos han sido resumidos por Anton Bertet (1973) y Guitián y Vázquez (1981), entre otros. Esencialmente siguen la siguiente secuencia al aumentar la temperatura:

- $100^{\circ} \mathrm{C}$. Pérdida de humedad.

- 300 a $500^{\circ} \mathrm{C}$. Destrucción de la materia orgánica presente.

- $550{ }^{\circ} \mathrm{C}$. Deshidroxilación de la caolinita y formación de metacaolinita.

- 600 a $800^{\circ} \mathrm{C}$. Transformación de los carbonatos.

- 900 a $1000^{\circ} \mathrm{C}$. Comienzo de la fusión de algunos minerales alcalinos, que termina a unos $1300^{\circ} \mathrm{C}$.

- $960^{\circ} \mathrm{C}$. Recristalización de la metacaolinita.

- 1000 a $1100{ }^{\circ} \mathrm{C}$. Destrucción de las micas presentes.

- $1100^{\circ} \mathrm{C}$ en adelante. Aparición de cristales de mullita.

La composición mineralógica encontrada en las distintas muestras presenta algunas variaciones en el contenido relativo de las fases principales identificadas, cuarzo, calcita, feldespatos (ortosa y plagioclasa), asi como en la presencia o no de algunos componentes minoritarios y trazas (mica, clorita, piroxeno, anfibol y menas metálicas). Como características texturales presentan en común una elevada porosidad y un tamaño relativamente grueso de los componentes mayoritarios en un amplio margen granulométrico.

Esta mineralogía revela una temperatura de cocción relativamente débil, inferior a la necesaria para la descomposición de los carbonatos (entre 600 y $800^{\circ} \mathrm{C}$ ) o para producir transfor- 
maciones apreciables en otros componentes. Desde el punto de vista mineralógico destaca igualmente la presencia de restos de anortosita en las muestras 1 y 4 entre los fragmentos adicionados a la pasta. Este hecho hace pensar que estas cerámicas sean de procedencia foránea, ya que no se encuentran rocas de este tipo a grandes distancias del templo de La Encarnación -al menos varios centenares de $\mathrm{km}$. que hayan podido emplearse como materia prima. No se puede afirmar lo mismo para las muestras de pebetero y exvoto, aunque tampoco se excluye que se trate de un material importado. Estos hechos parecen ser concluyentes para sospechar que las cerámicas descritas como pebetero y antefija se importaran ya elaboradas.

\section{BIBLIOGRAFIA}

ANTÓN BERTET, G., 1973: Análisis por difracción de rayos X de cerámicas ibéricas valencianas. SIP. Dip. Prov. Valencia, Ser. Trabajos n. ${ }^{\circ} 45$.

ARANA, R., 1985: Estudio mineralógico de unas cerámicas romanas de Aguilas y Mazarrón (Murcia). VI Congreso Internacional de Arqueologia Submarina, 443-447. Cartagena, 1982. Madrid.

BALCÁZAR, J. L.; Gerónimo, A., Y DE ANDRÉs, A., 1983: Reconocimiento mineralógico de cerámicas precolombinas. Bol. Soc. Española de Mineralogia, 7, 191-198.

BENNETT, H., Y OLIVER, G., 1992: XRF Analysis of Ceramics. Minerals and allied Materials. John Wiley \& Sons. Chichester, UK.

De ANDRÉs, A. M.; PALACIOS, J. M. Y MENÉndeZ, P., 1988: Characterization of Ceramic Sherds from the Period Transition of Bronze to Iron Ages in the Central Region of Iberian Peninsula. International Symposium on Archaeometry. Mayo. Toronto.

DE ANDRÉs, A. M.; PALACIOS, J. M., Y MENÉNDEZ, P., 1989: Estudio tecnológico de cerámicas grafitadas de la provincia de Guadalajara (periodo de transición de la Edad del Bronce a la Edad del Hierro). Bol. Soc. Española de Mineralogia. 12, 151-159.

Garcia Ramos, G.; Mesa, J. M.; Ruiz Abrio, M. T., y Sánchez Soto, P.J., 1986: Arcillas sericiticas de Garlitos (Badajoz): Mineralogía y propiedades cerámicas. Bol. Soc. Española de Mineralogia, 9, 175-183.

Guitian Rivera, F., Y VAzquez VARela, J. M., 1981: Sobre la tecnología de la cerámica castreña: Determinación de la temperatura de cocción. Rev. Arqueologia, 4, 89-93.

JOURDAIN, A., 1966: La technologie des produits ceramiques refractaires. Gauthiers-Villars. Paris. Singer, F., Y Singer, S. S., 1979: Cerámica Industrial, Vol. I. Urmo. Bilbao. 